\title{
Appearance deceives: unusual pneumothorax
}

\section{Traumatic phrenic nerve paralysis}

\author{
Paolo Gritti • Luigi Andrea Lanterna • \\ Carlo Brembilla $\cdot$ Ferdinando Luca Lorini
}

Received: 6 July 2014/ Accepted: 30 July 2014/Published online: 20 August 2014

(C) SIMI 2014

A 32-year-old motorcyclist was ejected a distance of several meters after a road accident. He was found unconscious, hypotensive $(90 / 50 \mathrm{mmHg})$, tachycardic (110 beats per minute) and dyspnoeic ( $\mathrm{SpO}_{2}$ of $92 \%$ in room air). During the initial assessment carried out by the emergency physician, an asymmetrical chest expansion was identified (Fig. 1a, Video 1S). Presuming a large pneumothorax, a small-caliber chest tube (8 French) was immediately inserted at the second intercostal space (Pleurocath ${ }^{\circledR}$, Plastimed Division, Prodimed ${ }^{\circledR}$, Le Plessis Bouchard, France) (Fig. 1b). Subsequently the patient was intubated and stabilized on a backboard with a cervical collar. When admitted to the Emergency Department (ED), during the initial neurological evaluation, the patient was still unconscious and maintained the chest wall asymmetry although the first X-ray of the chest performed in supine position was negative for evident pneumothorax. The physicians of the ED, presuming that the chest radiogram missed an anterior pneumothorax, and that the first drain was not working properly, decided to introduce on the midaxillary line another larger (28 French) drain (Argyle ${ }^{\mathrm{TM}}$, Coviden $^{\circledR}, 15$ Hampshire street, Mansfield, USA).

However, a subsequent CT scan of the chest did not show a pneumothorax or any rib fractures (Fig. $1 \mathrm{~b}$ and c).

Electronic supplementary material The online version of this article (doi:10.1007/s11739-014-1114-3) contains supplementary material, which is available to authorized users.

P. Gritti $(\bowtie)$ · F. L. Lorini

Department of Anaesthesia and Intensive Care, Ospedale Papa

Giovanni XXIII ${ }^{\circ}$, Bergamo, Italy

e-mail: grittip@libero.it

L. A. Lanterna $\cdot$ C. Brembilla

Department of Neuroscience and Neurosurgery, Ospedale Papa

Giovanni XXIII ${ }^{\circ}$, Bergamo, Italy
During the following days, the patient regained consciousness, but maintained an asymmetrical chest expansion and a left arm paralysis (Fig. 1d, e, Video 2S). A CT scan and MRI of the brain were negative, so an MRI of the spinal column was performed (Fig. 1f).

The MRI scan of the spinal column revealed the presence of pseudomeningoceles as a secondary injury to the traumatic nerve root avulsion, along the C1-C6 left vertebral column (Fig. 1f, Video 3S).

The case here concerns misinterpreting the unilateral paralysis of the diaphragm as a large pneumothorax. Symptoms of pneumothorax include dyspnoea, pleuritic chest pain, and if the pneumothorax is large, the affected side may be enlarged. With tension pneumothorax, hypotension can occur, however, there are other physical findings such as absent tactile fremitus, hyper-resonance to percussion, and decreased breath sounds on the affected side that can help to identify a pneumothorax. Chest radiography is currently considered an essential tool in confirming the diagnosis of pneumothorax; however, it misses $30-72 \%$ of pneumothoraces $[1,2]$. In particular, the antero-posterior supine projection may not be able to detect pneumothorax in traumatized patients because of the mandatory supine position, air in the pleural space migrates toward the anterior thoracic wall $[1,2]$. Pleural ultrasound imaging is more sensitive in the detection of pneumothorax with an anterior location $[1,2]$.

The phrenic nerve originates mainly from the fourth cervical nerve, but also receives contributions from the fifth and third cervical nerves [3]. The left arm and phrenic nerve paralysis resulted from an interruption of transmission of the nerve impulses through the brachial and phrenic nerves. Brachial plexus injuries occur in $1.2 \%$ of multitrauma patients, while phrenic nerve dysfunction is rarer $[4,5]$. Since accurate diagnosis of traumatic phrenic nerve 

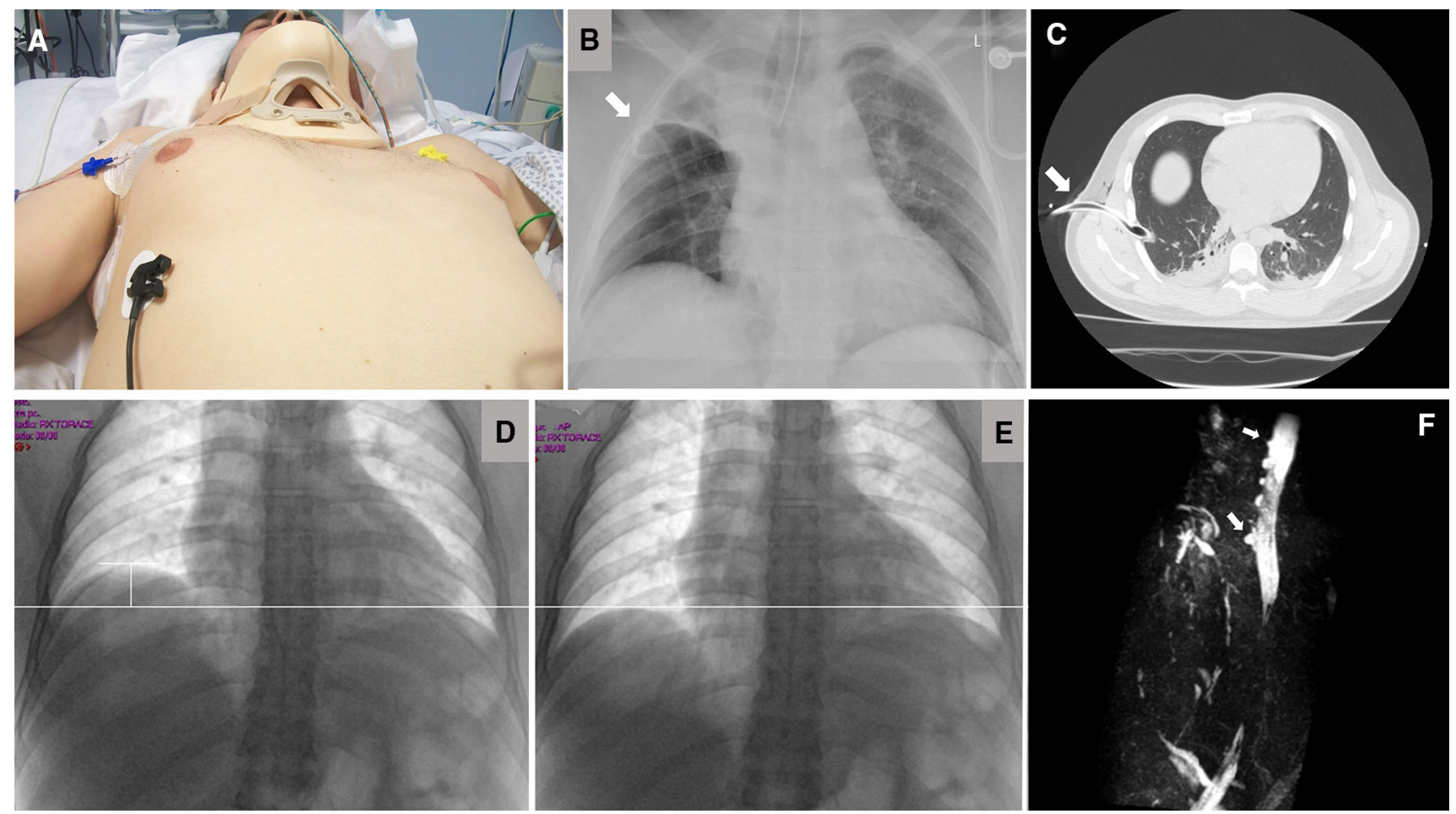

Fig. 1 Asymmetrical chest expansion (a). Chest radiograph (b) demonstrating the course of the first thoracostomy tube, and CT scan of the chest (c) showing the second thoracostomy tube (arrows). Note the apical atelectasis of the right superior lobe probably due to the low endotracheal tube, and the absence of subcutaneous emphysema and

paralysis in the emergency setting is often delayed or misinterpreted, it is prudent to look for findings that support the presence of a pneumothorax, such as subcutaneous emphysema, and rib fractures. In the initial chest X-ray study, the position is supine, there is no subcutaneous emphysema, and there are no rib fractures $[4,5]$.

\section{Conflict of interest None.}

\section{References}

1. Soldati G, Testa A, Pignataro G, Portale G, Biasucci DG, Leone A, Silveri NG (2006) The ultrasonographic deep sulcus sign in traumatic pneumothorax. Ultrasound Med Biol 32(8):1157-1163 rib fractures $(\mathbf{b}, \mathbf{c})$. Chest X-rays acquired in the expiratory (d) and inspiratory phase (e) of the respiratory cycle. Note the difference in excursion between the hemi-diaphragms in expiratory phase (d, e white lines). MRI scan of the spinal column shows the presence of pseudomeningoceles along the C1-C6 left vertebral column (f)

2. Soldati G, Testa A, Sher S, Pignataro G, La Sala M, Silveri NG (2008) Occult traumatic pneumothorax: diagnostic accuracy of lung ultrasonography in the emergency department. Chest 133(1):204-211

3. Williams PL, Warwick R, Dyson M, Bannister LH (1995) Gray's anatomy, 38th edn. Churchill Livingstone, Edinburgh

4. Midha R (1997) Epidemiology of brachial plexus injuries in a multitrauma population. Neurosurgery 40(6):1182-1188

5. Rhee PC, Pirola E, Hébert-Blouin MN, Kircher MF, Spinner RJ, Bishop AT, Shin AY (2011) Concomitant traumatic spinal cord and brachial plexus injuries in adult patients. J Bone Joint Surg Am 93(24):2271-2277 